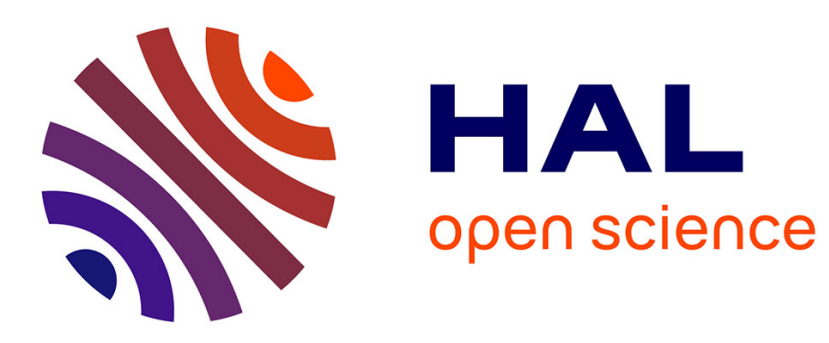

\title{
On Optimizing the Operating Conditions of a Mach-Zehnder Modulator for IR-UWB over Fiber Transmission
}

H Taki, S Azou, A Hamie, A Al Housseini, A Alaeddine, A Sharaiha

\section{- To cite this version:}

H Taki, S Azou, A Hamie, A Al Housseini, A Alaeddine, et al.. On Optimizing the Operating Conditions of a Mach-Zehnder Modulator for IR-UWB over Fiber Transmission. IEEE Int. Conf. on Digital Information and Communication Technology and its Applications, Apr 2015, Beyrouth, Lebanon. hal-01508682

\section{HAL Id: hal-01508682 \\ https://hal.science/hal-01508682}

Submitted on 14 Apr 2017

HAL is a multi-disciplinary open access archive for the deposit and dissemination of scientific research documents, whether they are published or not. The documents may come from teaching and research institutions in France or abroad, or from public or private research centers.
L'archive ouverte pluridisciplinaire HAL, est destinée au dépôt et à la diffusion de documents scientifiques de niveau recherche, publiés ou non, émanant des établissements d'enseignement et de recherche français ou étrangers, des laboratoires publics ou privés. 


\title{
On Optimizing the Operating Conditions of a Mach-Zehnder Modulator for IR-UWB over Fiber Transmission
}

\author{
H. Taki*†, S. Azou ${ }^{\dagger}$, A. Hamie ${ }^{* \ddagger}$, A. Al Housseini*, A. Alaeddine* and A. Sharaiha ${ }^{\dagger}$ \\ ${ }^{*}$ Lebanese University, Lebanon \\ ${ }^{\dagger}$ ENIB / CNRS UMR 6285 Lab-STICC, Brest, France \\ ${ }_{\ddagger}^{\ddagger}$ CRITC research center, Arts Sciences and Technology University in Lebanon (AUL)
}

\begin{abstract}
The power efficiency of a 2 Gbps OOK-based Impulse Radio-over-Fiber (RoF) system based on 5th-derivative Gaussian and Abraha's pulse is studied in this paper, with the aim of optimizing the Mach-Zehnder Modulator (MZM) setup. The best peak-to-peak (p-p) voltage is computed for several fiber lengths up to $40 \mathrm{Km}$. It is shown that adapting the p-p voltage with distance, with a trade-off between MZM output power and linearity while respecting FCC spectral constraints, can improve the system performance.
\end{abstract}

\section{INTRODUCTION}

As there is a growing demand for high speed and low power transmission systems nowadays, ultra wide band (UWB) radio technology becomes an important technique to be used in the new generation of short-range broadband wireless communications. The main advantages offered by UWB are the high data rate, immunity to multipath propagation, flexibility in reconfiguring data rate and power, very good time domain resolution, accurate mobile user location, and easy data protection [1], [2]. UWB applications include local and wide area networks, sensor networks, emergency communications, radar, remote sensing, and military applications. Over fiber transmission was a desirable solution to extend the UWB environment via optical access networks providing wide coverage area services [3]. Such infrastructures require direct optical pulse generation or electro-optical conversion utilizing intensity modulators [4]. Impulse radio ultra wide band (IR-UWB) technique has shown a great interest to be used due to its low complexity and cost transceiver architectures [5]. In 2002, the Federal Communication Commission (FCC) allowed UWB devices to operate at low power, within the so-called FCC mask, so as to avoid dangerous interference with other narrow band communication systems. Thus the transmitted power spectral density of UWB signals has to remain under the limit of $-41.3 \mathrm{dBm} / \mathrm{MHz}$ in the frequency range of [3.1-10.6] GHz. This power limitation has raised the need for highly efficient IR-UWB waveforms. In this paper, a MZM is considered at the transmitter and the trade-off between linearity and output power is investigated so as to cope with fiber properties. The best DC biasing voltage is known to be $V_{D C}=1.5 V_{\pi}$ ( $V_{\pi}$ being the halfwave voltage), corresponding to the most linear zone of MZM transfer function. Then, we study the impact of electrical IRUWB p-p driving voltage on the spectral efficiency of a 2 Gbps
OOK modulated signal after propagation over single mode fiber. As pulse waveforms, we consider the conventional 5thderivative Gaussian pulse together with Abraha's combination [6].

\section{RAdio Over Fiber System ARChitecture}

The Radio Over Fiber System (RoF) system considered here is illustrated in fig. 1; it consists of a MZM biased by the IRUWB signal generated electrically and fed by an input optical power produced from a laser diode. A Single Mode Fiber (SMF) is adopted for signal propagation, with a chromatic dispersion (CD) of $17 \mathrm{ps} / \mathrm{nm} / \mathrm{km}$ and an attenuation of 0.2 $\mathrm{dB} / \mathrm{km}$; a photo-detector then converts the received optical power into an electrical voltage signal to be attenuated before electromagnetic propagation through antenna. An attenuator is placed for stepping down the spectrum to meet FCC mask when the power spectral density exceeds regular limit.

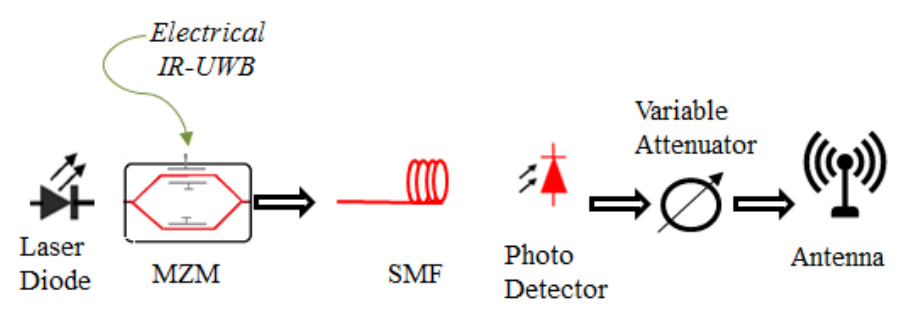

Fig. 1: Radio over Fiber system architecture.

The power of the laser diode is $P_{i n}=10 \mathrm{~mW}$ and the DC biasing voltage at the MZM is $V_{D C}=1.5 V_{\pi}$ with $V_{\pi}=6 \mathrm{~V}$. The laser diode, MZM, and SMF are implemented in Matlab. The photo-detector is assumed to be ideal, based on the instantaneous power obtained from optical field at SMF output. At MZM output, we can consider the standard expression for the optical power (injected into fiber):

$$
P_{\text {out }}(t)=P_{\text {in }} \cos ^{2}\left(\frac{\pi}{2 V_{\pi}}\left(V_{D C}+V_{p p} . s(t)\right)\right.
$$

where $s(t)$ denotes the normalized IR-UWB signal, such as $\max (|s(t)|)=1$. Figure 2 illustrates the MZM transfer function versus input voltage $V_{i n}$, including both DC component and modulating signal. 


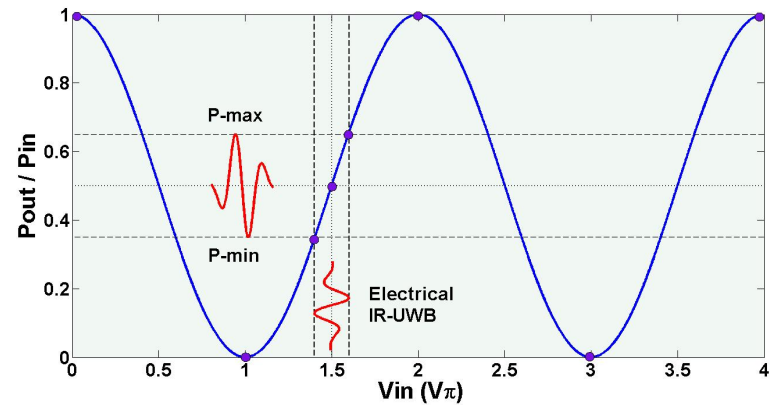

Fig. 2: MZM transfer function.

The DC component is removed prior to electromagnetic transmission, thus a compromise between the average power and linearity is required due to the nonlinear transfer function (1). So increasing the $\mathrm{p}-\mathrm{p}$ voltage adds strong nonlinearities to the input UWB pulse, but raising at the same time the extinction ratio (ER) $P_{\max } / P_{\min }$ and average output power. Larger ER translates into better bit error rate (BER) performance, as an energy detector-based non-coherent receiver is targeted. The average power directly influences the optical transmission reach that can be achieved, as no optical booster is placed before fiber. RoF networks with wide covering necessitates a large enough available power at the transmitter side, which corresponds to a large p-p voltage. On the other hand, free space transmission involves a strict power limitation imposed by the regulatory bodies (FCC mask), and MZM linearity becomes important to meet such constraint, which can be achieved by operating at small p-p voltages. Therefore, an optimization of MZM operating conditions has to be done for satisfying both constraints.

\section{MZM NON-LINEAR EFFECTS FOR SOME GAUSSIAN PULSES}

Before studying the MZM response versus input p-p driving voltage, we must choose the appropriate pulses to be used in terms of power efficiency and required system hardware complexity. The gaussians family is a pertinent solution, as some gaussians pulses can be generated using simple analog electronic components while offering very good spectral properties over the [3.1-10.6] GHz frequency band [7]. In our study we used the conventional 5th-derivative Gaussian pulse, together with a combination of delayed doublets recently proposed by Abraha [6].

The time waveforms and corresponding power spectral densities (PSDs) of the considered pulses are plotted in Figure 3. The analytical time expression of 5th-derivative Gaussian pulse $x_{5}(t)$ is described in (2), and equation (3) gives Abraha's pulse expression, from a linear combination of modified doublets $x_{2}(t)$ defined in (4). The pulse shaping factor $\sigma$ was chosen as follows: $\sigma=51 \mathrm{ps}$ for $x_{5}(t)$ and $\sigma=43 \mathrm{ps}$ for $x_{2}(t)$. The combination delay used for Abraha's pulse $x_{A}(t)$ is $\tau=25 \mathrm{ps}$, and the scaling factor associated with $x_{2}(t)$ is chosen as $k=1.17$.
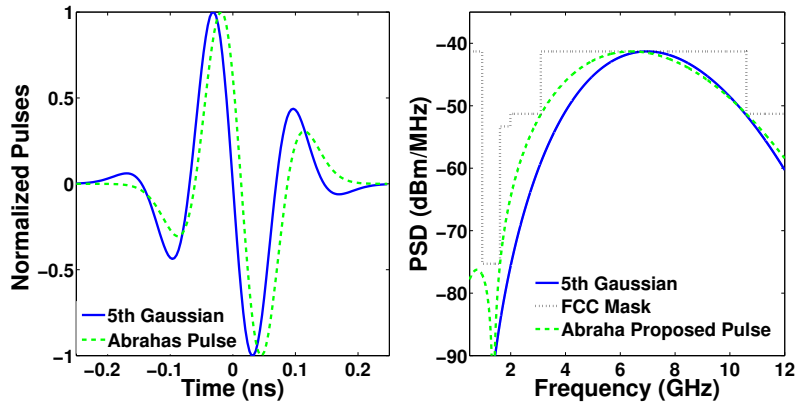

Fig. 3: Time waveforms and power spectral densities for pulses used in simulations.

$$
\begin{gathered}
x_{5}(t)=\left(\frac{-t^{5}}{\sigma^{4}}+\frac{10 t^{3}}{\sigma^{2}}-15 t\right) e^{\frac{-t^{2}}{2 \sigma^{2}}} \\
x_{A}(t)=x_{2}(t)-x_{2}(t-\tau) \\
x_{2}(t)=\left(\frac{-1}{\sigma^{2}}\right)\left(1-k \frac{t^{2}}{\sigma^{2}}\right) e^{\frac{-t^{2}}{2 \sigma^{2}}}
\end{gathered}
$$

For an OOK modulated signal $x_{O O K}(t)$ with a power spectral density $S_{O O K}(f)$, we have defined the criterion of power efficiency as being the collected power over [3.110.6] $\mathrm{GHz}$ for $x_{O O K}(t)$ over the maximum possible acquired power. As the sinc pulse achieves a full coverage over the entire UWB spectral mask (100\% efficiency), we considered a reference OOK-modulated signal $x_{O O K \text {,sinc }}(t)$ based on sinc. Hence, we defined the power efficiency as

$$
\eta_{O O K}=\frac{\int_{3.1 \mathrm{GHz}}^{10.6 \mathrm{GHz}} S_{\mathrm{OOK}}(f) d f}{\int_{3.1 \mathrm{GHz}}^{10.6 \mathrm{GHz}} S_{\text {OOK }, \text { Sinc }}(f) d f} \times 100 \%
$$

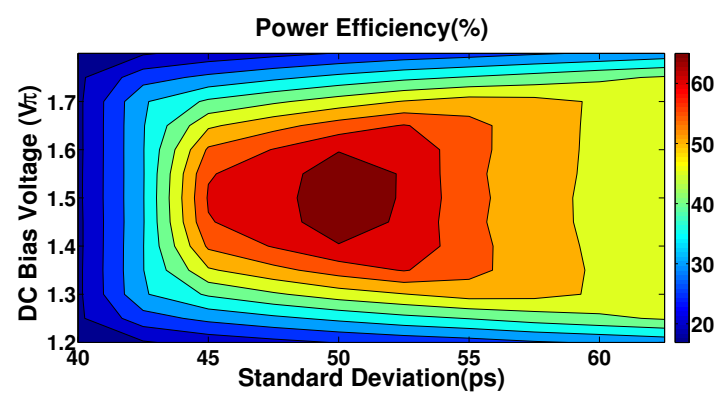

Fig. 4: Power efficiency versus pulse shaping factor and DC biasing voltage.

In fig. 4 we can observe the power efficiency at MZM output as a function of the applied DC bias voltage $V_{D C}$ and pulse shaping factor for the 5th-derivative Gaussian. Regarding the standard deviation $\sigma$, the best operating point lies at 51ps for which the spectrum peak is located at the midpoint of the UWB frequency interval, with a non-symmetrical response due to FCC mask shape. About the biasing voltage impact, the optimum efficiency is associated with the most linear region 
at $V_{D C}=1.5 V_{\pi}$, moving away from this point yields to a spectral distortion caused by the sharp curve of power ratio, the contour plot vertical symmetry is due to the cosine parity for MZM.
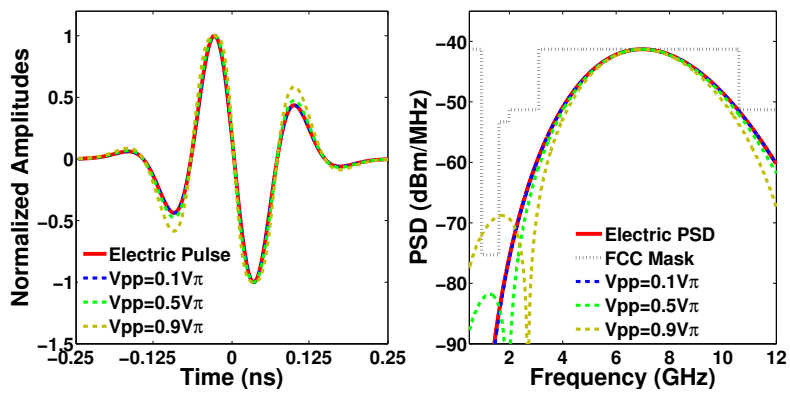

Fig. 5: Time waveforms and power spectral densities of 5thderivative gaussian pulse for several $\mathrm{p}-\mathrm{p}$ voltages.

Figure 5 shows the influence of p-p voltage $V_{p p}$ in time and frequency domain for 5 th-derivative gaussian pulse. As $V_{p p}$ increases more time spread is obtained, specially at positive and negative peaks, leading to low frequency components which violate FCC mask in the most restricted region [0.96-1.61] $\mathrm{GHz}$ corresponding to GPS band. This dangerous interference obliges the transmitter to step down the spectrum level so as to respect FCC limit, and consequently a low power efficiency is obtained.

\section{Optimal MZM PeaK-to-Peak Voltages}

Figure 6 shows the time waveforms of electrical OOK for a data sequence made of 8 bits '11010111', with the 5th-derivative gaussian and Abraha's pulse, respectively. All frames are periodically equal and for each symbol bit a single frame is transmitted (unit processing gain). The distortion of the received pulses at $40 \mathrm{Km}$, plotted in fig. 7, is due to the combined effects of MZM (driven by $V_{p p}=0.5 V_{\pi}$ ) and fiber $\mathrm{CD}$, which is also the main reason for imperfections existing in the ' 0 ' bit frames where no pulse is transmitted. Regarding MZM nonlinearities, applying large p-p voltage $\left(V_{p p}=0.9 V_{\pi}\right)$ leads to a strong spectral violation to $F C C$ at $5 \mathrm{~km}$ as shown in fig. 8. Hence, there is no benefit from high electrical power available as it is impossible to transmit an OOK with such a spectrum, unless the latter is adapted with standard limit. Whereas in the case of low p-p voltage $\left(V_{p p}=0.1 V_{\pi}\right)$ a regular spectrum is obtained, with no attenuation required for transmitting the overall stream of symbols. Note that the regularly spaced spectral lines result from the modulation process, the space between two consecutive lines corresponding to the data rate.

At $40 \mathrm{~km}$, a decrease in the spectrum levels is obtained (fig. 9) due to optical attenuation threw fiber. Then linearity is no longer important, as operating far below FCC mask raises the priority towards high average power instead of perfect spectral shape. Thus an increase in the p-p voltage is necessary to get a better performance at long distances. Therefore, the trade-off between linearity and output power depends on the
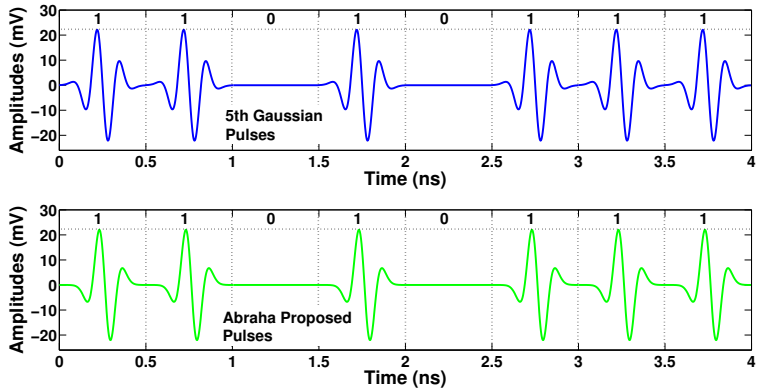

Fig. 6: Electrical OOK based on 5th-derivative gaussian pulse (above) and Abraha's pulse (below).
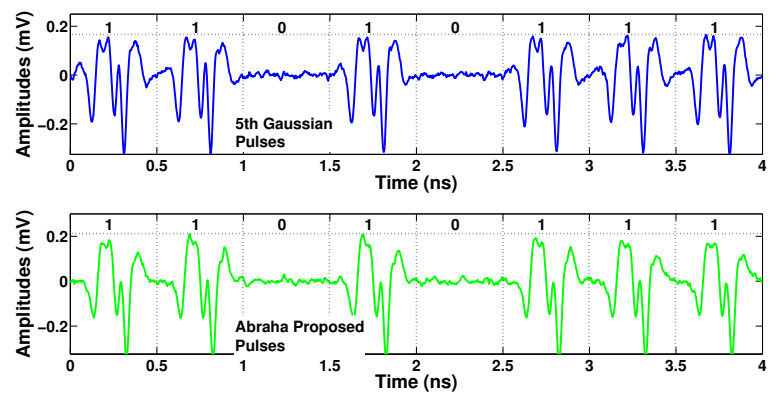

Fig. 7: Optical OOK at $40 \mathrm{Km}$ based on 5th-derivative gaussian pulse (above) and Abraha's pulse (below), with $V_{p p}=0.5 V_{\pi}$.

fiber length that have to be considered; the corresponding optimal p-p voltage at MZM input is investigated in the sequel.
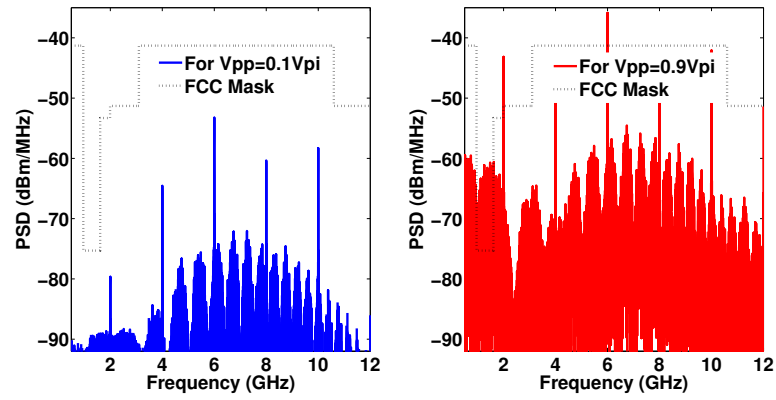

Fig. 8: OOK power spectral densities at $5 \mathrm{Km}$ for the case of $V_{p p}=0.1 V_{\pi}$ (left) and $V_{p p}=0.9 V_{\pi}$ (right).

The power efficiencies for the cases of using 5th-derivative Gaussian and Abraha's pulses are plotted in fig. 10 and fig. 11, respectively, for a wide range of p-p voltage and fiber length. The optimum performance is obtained in the interval $\left[0.3 V_{\pi}\right.$, $\left.0.5 V_{\pi}\right]$ at low distances for both cases, where no significant effects of dispersion/attenuation occur. As noticed, the contour plot is not symmetrical around the vertical line, so the axis of symmetry has a right deviation from the vertical direction, due to optical power degradation threw fiber which necessitates a compensation by increasing the p-p voltage. In fig. 12, some samples are re-plotted for a better indication at separate lengths of optical link. 

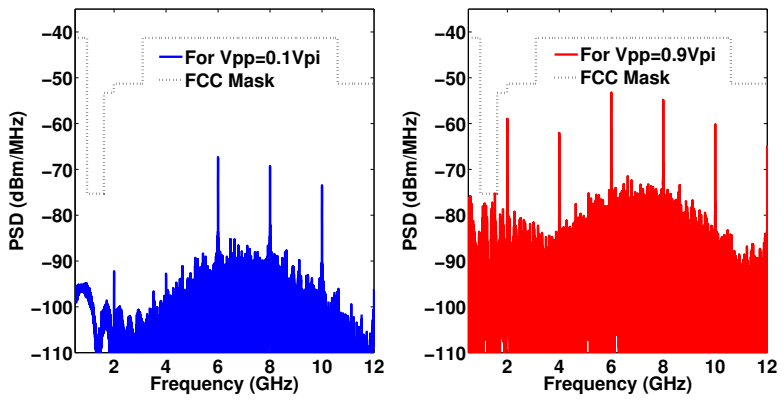

Fig. 9: OOK power spectral densities at $40 \mathrm{Km}$ for the case of $V_{p p}=0.1 V_{\pi}$ (left) and $V_{p p}=0.9 V_{\pi}$ (right).

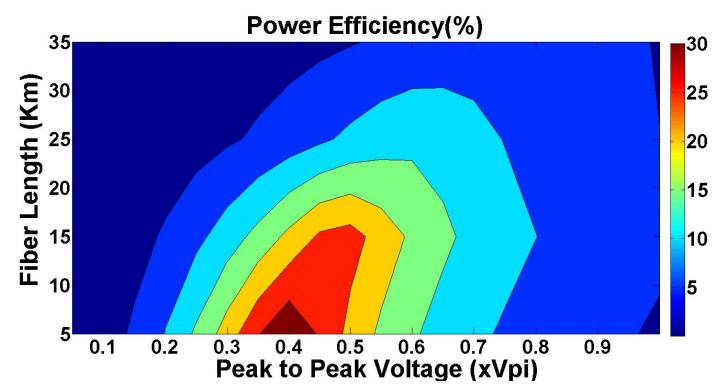

Fig. 10: Power efficiency versus fiber length and $p-p$ voltage for OOK based on 5th-derivative gaussian pulse.

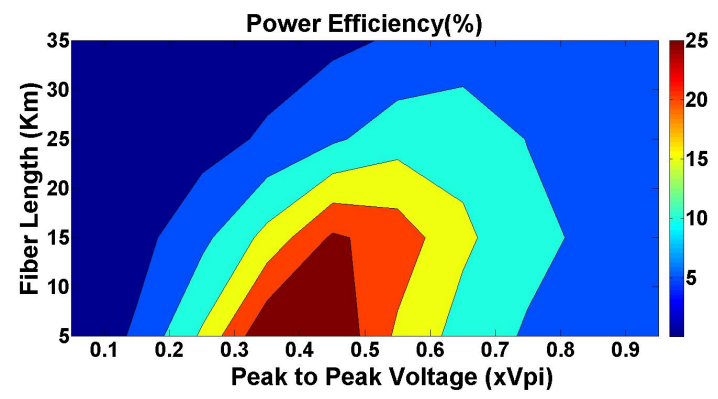

Fig. 11: Power efficiency versus fiber length and $p-p$ voltage for OOK based on Abraha's pulse.

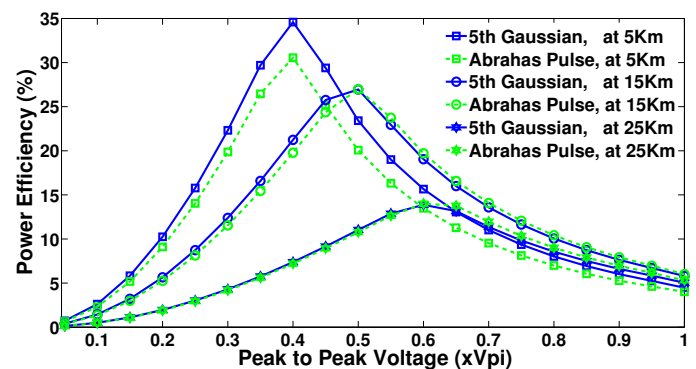

Fig. 12: Power efficiency versus $p-p$ voltage for several fiber lengths using 5th-derivative gaussian and Abraha's pulses.

The main conclusion of our study can be clearly observed in fig. 13 , where power efficiency is plotted versus SMF length. Following the traditional approach, having as the only interest the linearity, we could fix the p-p voltage at $V_{p p}=0.4 V_{\pi}$, which maximizes the efficiency at low fiber distances. But it is clear that keeping this particular value for operating over larger distances is no longer the best choice. Therefore, better performance does not necessarily mean high linearity for external modulation.

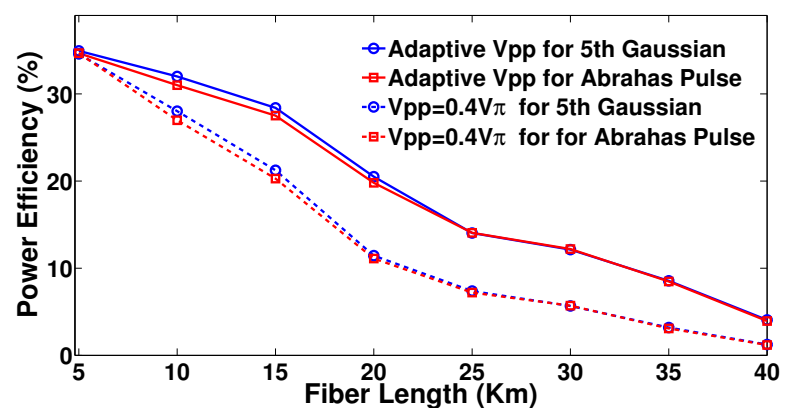

Fig. 13: Power efficiency versus fiber length for the cases of using fixed and optimal p-p voltages.

\section{CONCLUSION}

The optimal peak-to-peak voltage for MZM driven by a 2 Gbps OOK-modulated IR-UWB signal has been investigated in this paper, with respect to optical fiber length. By considering the criterion of power efficiency, it has been shown that use of the nonlinearities of the MZM may be beneficial for coping with fiber effects. Regarding pulse waveform, no clear advantage between the conventional 5th-derivative gaussian and Abraha's pulse has been observed if only the power efficiency is taken into account; however, we know that Abraha's pulse requires lower implementation complexity, so it should be adopted for practical applications. Placing a semi-conductor optical amplifier can be an interesting point to investigate in the future, with the aim of increasing the transmitted power and achieving a larger transmission reach.

\section{REFERENCES}

[1] M. G. Di Benedetto, "UWB communication systems: a comprehensive overview", (Vol. 5). Hindawi Publishing Corporation, 2006.

[2] G. R. Aiello, and G. D. Rogerson, "Ultra-wideband wireless systems", IEEE Microwave Mag., 4(2), pp. 36-47, 2003.

[3] F. Zeng, and J. Yao, "Ultrawideband impulse radio signal generation using a high-speed electrooptic phase modulator and a fiber-Bragg-gratingbased frequency discriminator", IEEE Photonics Technol. Letters, 18(19), pp. 2062-2064, 2006.

[4] M. Mirshafiei, A. Ghazisaeidi, D. Lemus, S. LaRochelle, and L. A. Rusch, "Upconversion of Gain-Switched Laser Pulses for Optical Generation of UWB Signals", J. Lightwave Technol., 30(2), pp. 207-214, 2012.

[5] Y. Park, and D. D. Wentzloff, "An all-digital $12 \mathrm{pJ} /$ pulse IR-UWB transmitter synthesized from a standard cell library", IEEE J. Solid-State Circuits, 46(5), pp. 1147-1157, 2011.

[6] S. T. Abraha, C. Okonkwo, P. A. Gamage, E. Tangdiongga, and T. Koonen, "Routing of power efficient IR-UWB wireless and wired services for in-building network applications", J. Lightwave Technol., 30(11), pp. 1651-1663, 2012

[7] H. Jin, L. Jiang, W. Hao, C. Sheng, H. Qijun, and Z. Yueping, "A CMOS fifth-derivative Gaussian pulse generator for UWB applications", J. Semiconductors, 35(9), pp. 1849-1852, 2014. 